\title{
Renovação das práticas editoriais e instruções aos autores da RBMFC a partir de 2019
}

\section{Renewal of editorial practices and author guidelines to RBMFC authors from 2019 \\ Renovación de las prácticas editoriales y directrices para autores de RBMFC a partir de 2019}

Leonardo Ferreira Fontenelle ${ }^{1}$, Thiago Dias Sarti ${ }^{1}[0$

${ }^{1}$ Revista Brasileira de Medicina de Família e Comunidade (RBMFC). Rio de Janeiro, RJ, Brasil.

\section{Resumo}

Neste editorial, são apresentadas as novas práticas editoriais e instruções aos autores que nortearão a Revista Brasileira de Medicina de Família e Comunidade (RBMFC) a partir de 2019. Com elas, a RBMFC renova seu compromisso com a publicação de trabalhos acadêmicos de qualidade para médicos de família e comunidade e outros profissionais da Atenção Primária à Saúde, contribuindo para a melhoria do cuidado à população.

Palavras-chave: Medicina de Família e Comunidade; Atenção Primária à Saúde; Publicações Periódicas; Comunicação Acadêmica; Políticas Editoriais

\begin{abstract}
This editorial presents the new editorial practices and author guidelines for Revista Brasileira de Medicina de Família e Comunidade (Brazilian Journal of Family and Community Medicine - RBMFC) starting in 2019. With them, RBMFC renews its commitment to publishing quality academic papers for family and community physicians and other primary care practitioners, contributing to the improvement of health care.
\end{abstract}

Keywords: Family Practice; Primary Health Care; Periodicals; Scholarly Communication; Editorial Policies

\section{Resumen}

Este editorial presenta las nuevas prácticas editoriales y las directrices para autores para la Revista Brasileira de Medicina de Família e Comunidade (Revista Brasileña de Medicina Familiar y Comunitaria - RBMFC) a partir de 2019. Con ellas, RBMFC renueva su compromiso de publicar documentos académicos de calidad para los médicos de familia y comunidad y otros profesionales de atención primaria, contribuyendo para la mejora de la atención de salud.

Palabras clave: Medicina Familiar y Comunitaria; Atención Primaria de Salud; Publicaciones Periódicas; Comunicación Académica; Políticas Editoriales

Como citar: Fontenelle LF, Sarti TD. Renovação das práticas editoriais e instruções aos autores da RBMFC a partir de 2019. Rev Bras Med Fam Comunidade. 2019;14(41):1963. https://doi.org/10.5712/rbmfc14(41)1963

\author{
Autor correspondente: \\ Leonardo Ferreira Fontenelle. \\ E-mail: leonardof@leonardof.med.br \\ Fonte de financiamento: \\ declaram não haver. \\ Parecer CEP: \\ não se aplica. \\ Procedência e revisão por pares: \\ não revisado por pares. \\ Recebido em: 04/02/2019. \\ Aprovado em: 04/02/2019.
}


A Revista Brasileira de Medicina de Família e Comunidade (RBMFC) é uma publicação da Sociedade Brasileira de Medicina de Família e Comunidade (SBMFC). Aparentemente redundante, essa definição é o fundamento da missão da RBMFC. De acordo com David Crotty, ${ }^{1}$ diretor editorial da Oxford University Press,

Toda sociedade com que já trabalhei se vê como a guardiã de seu campo. A sociedade tem como objetivo reunir e dar suporte a sua comunidade de pesquisa, promover o estudo do assunto que representa e direcionar o financiamento quando disponível, e guiar e proteger a integridade dessa pesquisa. Publicar uma revista é uma extensão natural dessa missão, colocando a expertise de seus membros a serviço em um processo de revisão por pares de alta qualidade para ajudar a aprimorar e dar visibilidade aos últimos achados de pesquisa. Revistas de sociedade são algumas vezes iniciadas para promover comunidades de pesquisa. Elas frequentemente não são apenas "uma revista", mas a saída para aquela comunidade de pesquisa e seus membros trabalhando no avanço de um campo específico. (tradução livre)

A medicina de família e comunidade (MFC) é uma especialidade relativamente jovem no Brasil e, graças a uma série de fatores, a SBMFC só está em funcionamento contínuo desde 2001 . $^{2}$ A RBMFC foi (re)lançada três anos depois, ${ }^{3}$ trazendo artigos que até hoje são referência, apesar da produção artesanal. $\mathrm{Na}$ época, a revista era publicada exclusivamente em formato impresso, de forma que as instruções aos autores eram resumidas em uma única página; os autores deveriam gravar seus artigos em um disquete e enviá-lo pelo correio. ${ }^{4}$ Nos anos seguintes, a produção editorial melhorou, as instruções aos autores passaram a se alinhar com normas internacionais, e os manuscritos passaram a ser recebidos por correio eletrônico e, depois, através da plataforma eletrônica livre Open Journal Systems (OJS), também conhecida no Brasil como Sistema Eletrônico de Editoração de Revistas (SEER). ${ }^{5}$ Originalmente distribuída em formato impresso para sócios da SBMFC e autores, a RBMFC passou a ser distribuída também em formato eletrônico, que em seguida se tornou o formato exclusivo de publicação. ${ }^{6,7}$

$\mathrm{Na}$ RBMFC, assim como em outras revistas científicas, as políticas editoriais e instruções aos autores se tornaram cada vez mais extensas e elaboradas, refletindo o espaço disponibilizado pelo formato eletrônico e o próprio amadurecimento das recomendações internacionais. Por exemplo, as recomendações do International Committee of Medical Journal Editors (ICMJE) ampliaram seu escopo da preparação do manuscrito ("Uniform Requirements for Manuscripts Submitted to Biomedical Journals") para todo o arco desde a condução da pesquisa até depois da publicação do artigo ("Recommendations for the Conduct, Reporting, Editing, and Publication of Scholarly Work in Medical Journals"). O estilo de formatação de referências "Vancouver" (cidade onde primeiro se reuniu o ICMJE) deixou de ser definido pelo ICMJE, e passou a ter como referência a National Library of Medicine dos Estados Unidos, ${ }^{8}$ organização responsável pela base de dados MEDLINE e pelo portal PubMed. Outro exemplo foram os Princípios de Transparência e Boas Práticas em Publicações Acadêmicas, ${ }^{9}$ publicados conjuntamente pelo Committee on Publication Ethics (Comitê de Ética em Publicações - COPE), o Directory of Open Access Journals (Diretório de Revistas de Acesso Aberto - DOAJ), a Open Access Scholarly Publishers Association (Associação de Editores Acadêmicos de Acesso Aberto - OASPA) e a World Association of Medical Editors (Associação Mundial de Editores Médicos - WAME). 
Ao longo de 2018, as políticas editoriais e instruções aos autores da RBMFC passaram por uma revisão completa, com o objetivo de torná-las mais claras e garantir o cumprimento das recomendações cabíveis. Essa revisão foi baseada não apenas nas duas recomendações mencionadas no parágrafo anterior, mas também nas Core Practices, do COPE;10 no White Paper on Promoting Integrity in Scientific Journal Publications, do Council of Scientific Editors; ${ }^{11}$ e nas diretrizes Transparency and Openness Promotion ${ }^{12}$ (em parte), além de outras mais específicas. Destacamos a seguir algumas das principais novidades:

- A redação da missão e escopo da revista passou a enfatizar mais claramente a MFC e a atenção primária à saúde (APS), estabelecendo a RBMFC como um meio de comunicação acadêmica entre pesquisadores e profissionais da APS/MFC.

- A política de seções passou a admitir explicitamente ensaios teóricos e metodológicos, revisões integrativas e de escopo, e relatos de experiência. Também esclareceu a diferença entre revisões clínicas ${ }^{13}$ e revisões baseadas na evidência, ${ }^{14}$ e consolidou algumas seções na nova seção Perspectivas.

- Foram estabelecidas as políticas de Conflitos de Interesse, Autoria e Colaboração, Ética em Pesquisa, e Dados Abertos e Reprodutibilidade, dando maior reconhecimento a orientações antes dispersas.

- Foram adotadas boas práticas como a declaração de contribuição de cada autor ou colaborador, a declaração de plano de compartilhamento de dados (obrigatória para ensaios clínicos) e a declaração do papel do financiador na condução da pesquisa.

- Os artigos passaram a ser publicados sob a licença Creative Commons Atribuição 4.0 Internacional.

Essa renovação das políticas editoriais e instruções aos autores é parte de um processo mais amplo, que é o permanente compromisso da RBMFC com o aprimoramento de suas práticas editoriais. As novas políticas editoriais e instruções aos autores se tornaram efetivas no fim de dezembro de 2018, e devem refletir-se progressivamente nos artigos publicados ao longo de 2019. Paralelamente, a RBMFC lançou uma chamada para editores associados, com vistas a recompor e dinamizar seu corpo editorial. Com relação aos avaliadores ad hoc, desde 2017 a RBMFC publica anualmente um agradecimento a quem efetivamente avaliou manuscritos naquele ano, em vez de simplesmente publicizar seu cadastro de avaliadores. Por fim, no início de 2018 a RBMFC assumiu um compromisso com a agilidade na avaliação e publicação de artigos. De fato, o tempo de avaliação dos artigos em geral foi inferior a dois meses, e o tempo até a publicação foi inferior a seis meses. Tudo isso sem abrir mão do rigor: cerca de metade das submissões foram declinadas sem avaliação por pares, e três quartos das submissões avaliadas por pares foram rejeitadas.

Em suma, a RBMFC reafirma seu compromisso com a promoção do intercâmbio acadêmico entre pesquisadores e profissionais, adotando boas práticas editoriais para publicar material relevante para a APS/MFC. 


\section{Conflito de interesses}

\section{Declaram não haver.}

\section{Referências}

1. Crotty D. Why Society and Not-For-Profit Journals Are Worth Preserving: Better Economic and Continuing Value for the Community [Internet]. The Scholarly Kitchen. 2018. [citado 2019 Jan 29]. Disponível em: https://scholarlykitchen.sspnet.org/2018/12/06/why-societyand-not-for-profit-journals-are-worth-preserving-better-economic-and-continuing-value-for-the-community/

2. Falk JW. A Medicina de Família e Comunidade e sua entidade nacional: histórico e perspectivas. Rev Bras Med Fam Comunidade. 2004;1(1):5-10. https://doi.org/10.5712/rbmfc1(1)2

3. Anderson MIP, Falk JW. Editorial. Rev Bras Med Fam Comunidade. 2004;1(1):1. https://doi.org/10.5712/rbmfc1(1)1

4. Wagner HL. Instruções para colaboradores da Revista da Sociedade Brasileira de Medicina de Família e Comunidade. Rev Bras Med Fam Comunidade. 2004;1(1):2.

5. Demarzo MMP, Savassi LCM, Milhomens DM, Gusso GDF. Nova etapa da RBMFC. Rev Bras Med Fam Comunidade. 2011;6(18):7-8. https://doi.org/10.5712/rbmfc6(18)297

6. Demarzo MMP, Milhomens DM. RBMFC em 2011: evolução e desafios. Rev Bras Med Fam Comunidade. 2011;6(21):227-9. https://doi. org/10.5712/rbmfc6(21)499

7. Gusso G, Poli P. A medicina de família e comunidade revista. Rev Bras Med Fam Comunidade. 2016;11(38):1-3. https://doi.org/10.5712/ rbmfc11(38)1319

8. Patrias K. Citing medicine: the NLM style guide for authors, editors, and publishers [Internet]. 2a ed. Bethesda (MD): National Library of Medicine (US); 2007. Disponível em: http://www.nlm.nih.gov/citingmedicine

9. Committee on Publication Ethics, Directory of Open Access Journals, Open Access Scholarly Publishers Association, World Association of Medical Editors. Princípios de Transparência e Boas Práticas em Publicações Acadêmicas [Internet]. 2018 Jan [citado 2019 Jan 29 ]. Disponível em: https://publicationethics.org/resources/translated-resources/princ\%C3\%ADpios-de-transpar\%C3\%AAncia-e-boaspr\%C3\%A1ticas-em-publica\%C3\%A7\%C3\%B5es-acad\%C3\%AAmicas

10. Committee on Publication Ethics. Core practices [Internet]. Committee on Publication Ethics: COPE. [citado 2019 Jan 29]. Disponível em: https://publicationethics.org/core-practices

11. Council of Science Editors, Editorial Policy Committee. CSE's White Paper on Promoting Integrity in Scientific Journal Publication [Internet] Wheat Ridge (CO): Council of Science Editors; 2018. Disponível em: https://www.councilscienceeditors.org/wp-content/uploads/CSEWhite-Paper_2018-update-050618.pdf

12. Nosek BA, Alter G, Banks GC, Borsboom D, Bowman SD, Breckler SJ, et al. Promoting an open research culture. Science. 2015;348(6242):1422-5. https://doi.org/10.1126/science.aab2374

13. Fontenelle LF, Brandão DJ. Uma proposta metodológica para a elaboração de revisões clínicas. Rev Bras Med Fam Comunidade. 2018;13(40):1-10. https://doi.org/10.5712/rbmfc13(40)1871

14. Braga R, Melo M. Como fazer uma revisão baseada na evidência. Rev Port Med Geral Fam.2009;25(6):660-6. https://doi.org/10.32385/ rpmgf.v25i6.10691 